\title{
Physicochemical characterization of engineered nanoparticles under physiological conditions: Effect of culture media components and particle surface coating
}

\author{
Revised and resubmitted to: \\ Colloids and Surfaces B: Biointerfaces \\ September 25, 2011 \\ JULIEN FATISSON ${ }^{1}$, IVAN R. QUEVEDO ${ }^{1}$, KEVIN J. WILKINSON ${ }^{2}$ and NATHALIE \\ TUFENKJI ${ }^{*}, 1$ \\ ${ }^{1}$ Department of Chemical Engineering, McGill University, \\ Montreal, Quebec H3A 2B2, Canada \\ ${ }^{2}$ Department of Chemistry, University of Montreal, \\ Montreal, Quebec H3C 3J7, Canada
}

* Corresponding Author. Phone: (514) 398-2999; Fax: (514) 398-6678; E-mail: nathalie.tufenkji@mcgill.ca 


\begin{abstract}
The use of engineered nanoparticles (ENPs) in commercial products has increased substantially over the last few years. Some research has been conducted in order to determine whether or not such materials are cytotoxic, but questions remain regarding the role that physiological media and sera constituents play in ENP aggregation or stabilization. In this study, several characterization methods were used to evaluate the particle size and surface potential of 6 ENPs suspended in a number of culture media and in the presence of different culture media constituents. Dynamic light scattering (DLS) and fluorescence correlation spectroscopy (FCS) were employed for size determinations. Results were interpreted on the basis of ENP surface potentials evaluated from particle electrophoretic mobilities (EPM). Measurements made after $24 \mathrm{hrs}$ of incubation at $37^{\circ} \mathrm{C}$ showed that the cell culture medium constituents had only moderate impact on the physicochemical properties of the ENP, although incubation in bovine serum albumin destabilized the colloidal system. In contrast, most of the serum proteins increased colloidal stabilization. Moreover, the type of ENP surface modification played a significant role in ENP behavior whereby the complexity of interactions between the ENPs and the medium components generally decreased with increasing complexity of the particle surface. This investigation emphasizes the importance of ENP characterization under conditions that are representative of cell culture media or physiological conditions for improved assessments of nanoparticle cytotoxicity.
\end{abstract}

Keywords: Nanoparticles, Cell culture media, Dynamic Light Scattering, Fluorescence Correlation Spectroscopy, Transmission Electron Microscopy, Zeta-potential. 


\section{Introduction}

Engineered nanoparticles (ENPs) have a heightened reactivity as well as distinctive magnetic and optical properties that make them ideal for numerous applications in the medical, food, cosmetic, and electronic industries [1-4]. Nonetheless, there is an increasing cause for concern, given their potential to penetrate cell membranes and cause adverse biological effects [58].

Several in vitro studies conducted with different cell lines have tracked the cytotoxicity of various ENPs including silica [9]; quantum dots [10, 11]; gold nanorods [12] or polystyrene nanospheres with cationic and anionic groups [13]. In all the studies, a direct correlation was shown between particle properties (surface charge, specific surface area, size or hydrophobicity) and their bioavailability. Unfortunately, comparisons among toxicity studies are often difficult or impractical due to differences in the cell lines that were tested, exposure times, particle concentrations or the choice of ENPs [6]. Hence, there is a continuing need to better understand the dynamic interactions among nanoparticles, the culture medium and cell surfaces [14].

Previous reports have investigated the effects of bovine serum albumin (BSA) or fetal bovine serum (FBS) on the size and surface potential of $\mathrm{TiO}_{2}$ and $\mathrm{ZnO}$ nanomaterials [14-16] and special attention has been paid to the role of surface coatings [16, 17]. Researchers have highlighted the importance of using several techniques in parallel to study protein-nanoparticle interactions [18]. Nonetheless, to date, we do not yet have a good mechanistic understanding of how the different components of biological media (e.g., proteins, salts, carbohydrates or serum) can interact and affect the stability of ENPs. In culture media, the properties of the ENP, such as surface charge and aggregation state, will be largely determined by the physicochemical properties of the solution (e.g., ionic strength, $\mathrm{pH}$, temperature). For example, through charge screening, the 
elevated ionic strength $(I \sim 150 \mathrm{mM})$ of biological fluids can decrease the electrostatic repulsive forces among surfaces, thereby increasing ENP aggregation [19] or increasing the attachment of the ENP onto biological surfaces. Furthermore, the adsorption of proteins onto ENP surfaces has been shown to result in the stabilization of colloidal suspensions [20, 21]. In that case, serum protein (e.g., albumin or fibrinogen) may adsorb onto the ENP surface, creating an interface known as a protein "corona", where the degree and kinetics of association will depend on the binding affinity and concentration of each protein [14]. Because the "corona" could interact both with the cell membrane and the ENP, it is essential to its interactions and effects on the ENPs [20].

In this study, we show the impact of $I$ and the adsorption of selected cell culture media components on the size and EPM of six ENPs: silica nanoparticles $\left(n \mathrm{SiO}_{2}\right)$, gold nanoparticles $(n \mathrm{Au}), \mathrm{CdTe}$ quantum dots (QD), carboxymethylcellulose-coated (CMC-nZVI) and bare zerovalent iron $(n \mathrm{ZVI})$ and carboxylated polystyrene latex nanospheres $(n \mathrm{PL})$. Particle stability was monitored in phosphate buffered saline (PBS), PBS with sodium pyruvate, L-glutamine, BSA or FBS, and two common cell culture media (DMEM and F-12K). Three different techniques were used to evaluate the size of the ENP: dynamic light scattering (DLS), fluorescence correlation spectroscopy (FCS) and transmission electron microscopy (TEM), while laser Doppler velocimetry was employed to measure the electrophoretic mobility (EPM). The factors that influence the interaction of the nanoparticles and the media components are examined in an effort to determine to what extent their interactions might influence in vitro studies of cytotoxicity. 


\section{Materials and methods}

\subsection{Nanoparticle sample preparation}

The cell culture media contain: amino acids (e.g. L-glutamine), carbohydrate sources (e.g. glucose, sodium pyruvate) or inorganic salts (e.g. PBS) and they are commonly supplemented with proteins (e.g. FBS and BSA) or hormones [22] (Table S1). To better understand the effects of the medium components on ENP stability, a number of increasingly complex media were prepared in deionized (DI) water (Table 1). Also, two cell culture media commonly used in nanoparticle cytotoxicity studies, i.e. Dulbecco's modified essential medium (DMEM, Invitrogen) and F-12K medium (Invitrogen), were evaluated, with or without the addition of $10 \%$ FBS.

\section{[TABLE 1 HERE]}

The ENPs selected for this study are among the nanomaterials listed by the Organization for Economic Cooperation and Development (OECD) as reference materials to support measurement, toxicology and risk assessment studies [23], excluding latex nanospheres ( $n \mathrm{PL})$ which have been used as a well-defined model particle to compare results. Bare $n \mathrm{ZVI}$ and CMC$n Z \mathrm{VI}$ particles were synthesized as previously described [24] and found to have nominal sizes of 77 and $94 \mathrm{~nm}$, respectively, using TEM [24]. All other particles were used as received by the manufacturer: $n \mathrm{SiO}_{2}(70 \mathrm{~nm}$ nominal size, Nissan Chemical Industries Ltd, TX, USA), carboxylated $n \mathrm{PL}$ (24 nm nominal size, Invitrogen), citrate stabilized $n \mathrm{Au}$ (10 $\mathrm{nm}$ nominal size, Sigma-Aldrich) and CdTe QD (10 nm nominal size, ViveNano). The ENPs can be broadly divided into 3 categories: (1) non-coated ENPs: $n$ Au and nZVI; (2) carboxyl-functionalized ENPs: CMCnZVI and $n \mathrm{PL}$, where the carboxyl groups are covalently bound to the ENP surface; and (3) polymer-embedded ENPs: QD and $n \mathrm{SiO}_{2}$, are stabilized with a physically bound polymer coating, 
whose detailed formulations were not disclosed by the suppliers. Prior to their dilution into the experimental media, $n \mathrm{SiO}_{2}, n \mathrm{Au}$, and QD stock suspensions were vortexed at high speed for 30 sec. The $n \mathrm{PL}$ suspension was sonicated for $5 \mathrm{~min}$ and the CMC- $n \mathrm{ZVI}$ and $n \mathrm{ZVI}$ suspensions were sonicated for 30 min. Stock suspensions were diluted in the experimental media at a final concentration of $1.4 \times 10^{12}$ particles $/ \mathrm{mL}$ for $n \mathrm{SiO}_{2}, n \mathrm{PL}$ and QD and $1.4 \times 10^{10}$ particles $/ \mathrm{mL}$ for $n \mathrm{Au}$, and $n \mathrm{ZVI}$ (due to the lower concentration of available stock suspension). ENP suspensions were vortexed at high speed for $30 \mathrm{sec}$ followed by equilibration at $37^{\circ} \mathrm{C}$ for $24 \mathrm{hrs}$ and then gently mixed by manual inversion prior to measurements.

\subsection{Electrokinetic characterization}

A Zetasizer Nano ZS (Malvern Instruments, UK) was used to measure the electrophoretic mobility (EPM) by laser Doppler velocimetry. All analyses were performed in triplicate, using three different samples (prepared on different days). A disposable folded capillary cell (DTS 1060) was used with an automatically-applied electrical field $(E)$ between 10 to $50 \pm 0.1 \mathrm{~V} / \mathrm{m}$. Measurements were acquired at $37.0 \pm 0.2{ }^{\circ} \mathrm{C}$.

\subsection{Size analysis}

Adequate nanoparticle sizing requires the use of multiple complementary characterization techniques [25]. The hydrodynamic diameter was estimated using a Zetasizer Nano ZS with a He$\mathrm{Ne} 633 \mathrm{~nm}$ red laser. Correlation functions were interpreted as a number-averaged particle size using the DTS Software (v.6.01). Measurements were carried out in triplicate, using three different samples prepared on three different days at $37.0 \pm 0.2^{\circ} \mathrm{C}$. 
Hydrodynamic diameters were also determined by means of fluorescence correlation spectroscopy [25-27] using a Leica TCS SP5 laser scanning microscope with an Ar ion laser for excitation $(514 \mathrm{~nm})$. Non-fluorescent ENPs $\left(n \mathrm{SiO}_{2}, n \mathrm{Au}, n \mathrm{ZVI}\right.$ and CMC- $\left.n \mathrm{ZVI}\right)$ were labelled by adding a small quantity $\left(10^{-8} \mathrm{M}\right)$ of rhodamine $6 \mathrm{G}$ (R6G, Sigma-Aldrich) prior to measurements. The concentration of R6G corresponded to $<0.1 \%$ nanoparticle surface coverage. An optimized setting time of 30-100 sec was used for data acquisition and measurements were repeated 10 times. In order to obtain the nanoparticle diffusion coefficients, the dimensions of the confocal volume were first calibrated using rhodamine 110 (Sigma-Aldrich), which has a known diffusion coefficient of $4.7 \times 10^{-10} \mathrm{~m}^{2} / \mathrm{s}$ in water [28]. Weight averaged diffusion coefficients were determined from the measured diffusion times through the calibrated confocal volume. FCS measurements were conducted at $22 \pm 1^{\circ} \mathrm{C}$ using at least three independent samples. Because it was not possible to conduct FCS measurements at $37^{\circ} \mathrm{C}$, these data are included here solely as supporting data for the DLS measurements. Furthermore, given that the characterization of QD has been known to lead to inaccurate size measurements by FCS due to their blinking [25], they were not performed here.

Bathochromic shifts in UV absorbance have been attributed to a change in the dielectric environment of metal-based nanoparticles [29] which might result from particle aggregation [30, 31]. UV-Visible spectra were recorded for $n \mathrm{Au}$ colloidal solutions with a UV/visible spectrophotometer (Hewlett-Packard Model 8453) using a $1 \mathrm{~cm}$ quartz cell with a $1 \mathrm{~mm}$ aperture.

Finally, Transmission Electron Microscopy (TEM) was used to obtain high-resolution images of two of the nanoparticles ( $n \mathrm{Au}$ and $\mathrm{QD}$ ) under selected conditions. The specimens were prepared following the procedure recommended by Mavrocordatos et al. [32]. In brief, a small droplet $(20 \mu \mathrm{L})$ of the nanoparticle suspension was placed onto Cu TEM grids (SPI Supplies) 
coated with a carbon-free $\mathrm{SiO}$ film then left to dry overnight. The images were taken with a Philips CM200 TEM equipped with a 2k×2k CCD camera (Advanced Microscopy Techniques Corp., MA, USA) and operating at $200 \mathrm{kV}$ with a $\mathrm{LaB}_{6}$ filament. Mean sizes and $95 \%$ confidence intervals were determined from the measurement of at least 10 particles per frame, in at least three randomly selected images recorded at high magnification $(>100000 \times)$.

\subsection{Statistical significance}

All results were presented as the mean of the assessed EPM or particle size for at least three independent measurements with the error bars representing the respective $95 \%$ confidence intervals. The difference between each experimental condition and the corresponding control for all ENPs was evaluated using Student $t$-tests. Symbols “*” or " $\star$ ” and “+” or " $x$ " have been employed to denote the statistically significant difference $(p<0.05)$ between values of size or EPM values with their corresponding control values, respectively (i.e. when no symbol is present, there is no significant difference between size and EPM values with the control values).

\section{Results}

Because numerous conditions and ENPs have been examined, in the following discussion, they have been ordered with respect to an increasing complexity of the medium "A to M" (Table 1) and increasing complexity of surface coating on the ENP (particles $i$ to vi, Figure 1).

\subsection{Characterization of non-coated ENPs in aqueous suspensions and effect of media composition} on particle stability 
For all of the investigated particles, EPM values (Figure 1) were negative over the entire range of studied conditions. Although the $n$ Au used here is stabilized with citrate (Figure $\mathrm{S} 1$ ), this ENP is discussed alongside the $n Z \mathrm{VI}$ due to the fact that citrate is a relatively small molecule compared to the other polymeric coatings (such as CMC) used to stabilize the other ENPs we studied. For the $n Z \mathrm{VI}$, measured sizes were significantly larger (3-4 times their nominal sizes) than the values obtained by TEM $(77 \pm 15 \mathrm{~nm})$ [24]. Indeed, even at low $I$, DLS values of $\sim 350 \mathrm{~nm}$ in $1 \mathrm{mM}$ $\mathrm{NaHCO}_{3}$ strongly suggest the formation of particle aggregates in this medium (probably caused by attractive magnetic forces). Suspensions of the $n \mathrm{Au}$ appeared to be fairly stable at low $I$ even though the nominal size reported by the manufacturer $(8.5-12 \mathrm{~nm})$ was significantly smaller than the results obtained by DLS $(\sim 30 \mathrm{~nm}$, about 3 times the nominal size) (Figure 1ii).

\section{[FIGURE 1 HERE]}

When the $I$ of the medium increased (medium A to medium B), sizes of $n Z \mathrm{VI}$ and $n \mathrm{Au}$ doubled or tripled, even though variations in EPM were negligible. The addition of sodium pyruvate (medium C) or L-glutamine (medium D) to the PBS medium (B) had relatively little effect on the EPM of the ENPs, but both pyruvate and L-glutamine resulted in increased aggregation of the $n \mathrm{Au}$ (2-fold and 5-fold increase in diameters, respectively) and no significant effect on the $n Z \mathrm{VI}$. To corroborate the observed phenomenon, $\mathrm{UV}$-visible spectra of diluted $n \mathrm{Au}$ suspensions were recorded in water and in pyruvate solution (C). The addition of this constituent resulted in a bathochromic shift (Figure 2), consistent with particle aggregation as also observed in other reported studies $[30,31]$.

[FIGURE 2 HERE] 
The addition of a small amount of albumin (medium E) did not cause a significant change in either the EPM or the sizes of $n \mathrm{ZVI}$ or $n \mathrm{Au}$ as compared to PBS (medium B). In contrast, a larger addition of albumin (10\%, medium F) promoted significantly greater aggregation of the $n \mathrm{Au}$ as compared to either the non-supplemented medium (B) or the medium with lower albumin concentration. The addition of FBS (media $\mathrm{G}$ and $\mathrm{H}$ ) resulted in increased colloidal stability of $n \mathrm{Au}$ as compared to both the control medium (B) and the media containing BSA (E and F). On the other hand, for the $n \mathrm{ZVI}$, only the addition of pyruvate may have increased particle stability, consistent with a more negative particle charge. Additions of L-glutamine (medium D), low concentration of BSA (medium E) or FBS (medium G) had negligible effect on $n$ ZVI size or EPM.

The above characterization of ENPs in suspension helps to enhance our understanding of nanoparticle behaviour in complex cell culture media (DMEM or F-12K, either in the presence or absence of supplements). In general, EPM values were quite similar in the four media ( $\mathrm{J}$ to $\mathrm{M}$ ). For the $n \mathrm{Au}$, sizes measured in DMEM (medium J) or F-12K (medium L) indicated clear evidence of ENP destabilization, with particle diameters that were several fold larger than either the bicarbonate (A) or PBS (B) controls. In contrast, the addition of 10\% FBS (media H, K, M) once again had a similar strongly stabilizing effect. Increased aggregation was observed for $n Z \mathrm{VI}$ in both the F-12K and DMEM media and the addition of FBS significantly decreased aggregation.

3.2. Characterization of carboxylated ENPs in aqueous suspensions and effect of media composition on particle stability

The CMC- $n \mathrm{ZVI}$ and $n \mathrm{PL}$ may be schematically represented as having short polymer brushes with negatively charged carboxylic groups at the ENP surface (Figures 1iii and 1iv 
respectively). Globally, these ENPs were much more stable in the experimental media than were the bare $n \mathrm{ZVI}$ and the $n \mathrm{Au}$. In medium $\mathrm{A}$, the measured diameters for $n \mathrm{PL}$ and CMC- $n \mathrm{ZVI}$ were close to the nominal particle sizes, indicating the absence of aggregation at low $I$. The increase of $I$ in medium B had little effect on the size of the $n$ PL, whereas DLS indicated a doubling of the CMC-nZVI size. While slight aggregation may have occurred in media C and D for the CMC$n Z \mathrm{VI}$, the addition of sodium pyruvate and L-glutamine did not appear to influence the measured sizes of the $n \mathrm{PL}$.

The addition of $0.1 \%$ BSA (medium E) had little effect on particle sizes or EPM values with respect to the control (medium B), however when the BSA proportion was increased to $10 \%$ (medium F), a significant increase of ENP size was observed for both the CMC-nZVI (300\% increase) and the $n \mathrm{PL}(1700 \%$ increase). These results strongly suggested that the albumin could adsorb on the surface of the carboxylated ENPs and promote particle heteroaggregation, as observed with the relatively uncoated ENPs ( $n \mathrm{Au}$ and $n \mathrm{ZVI})$. As above, CMC- $n \mathrm{ZVI}$ and $n \mathrm{PL}$ aggregated significantly in complex cell culture media (media $\mathrm{J}$ and L) whereas all media supplemented with serum (media G, H, K and M) showed significant colloidal stability when compared to their respective controls.

\subsection{Characterization of polymer-embedded ENPs in aqueous suspensions and effect of media composition on particle stability}

The addition of polymers to colloidal suspensions has been found to greatly improve their stability $[17,19,33]$. Based on the information provided by the $n \mathrm{SiO}_{2}$ and QD suppliers, the polymers used in manufacturing these particles were of relatively high molecular weight and 
physically adsorbed at the surface of the ENP. Indeed, particle sizes obtained by DLS in the low I media were only slightly larger than sizes reported by the manufacturer, indicating stable suspensions of individual $n \mathrm{SiO}_{2}$ and QD particles.

The effects of the different cell culture constituents were fairly consistent with the results obtained for the other ENPs. The presence of even low concentrations of BSA promoted aggregation of these ENPs (media E and F) whereas FBS was again observed to stabilize the colloidal suspensions (media $\mathrm{G}, \mathrm{H}, \mathrm{K}$ and $\mathrm{M}$ ). Unlike the CMC-nZVI and $n \mathrm{PL}$, the polymerembedded ENPs were stable even when added to the serum-free culture media (J, L).

\subsection{Characterization of ENPs in aqueous suspensions by complementary techniques}

The use of DLS has been often criticized since the scattering intensity of the particles (roughly proportional to $d^{6}$ ) skews the interpretation of particle size towards larger particles in the analysis of polydisperse colloidal systems. In FCS, the signal scales with $d^{4}$ [27] resulting in an analytical signal that is less affected than DLS by the presence of particle aggregates [25]. TEM, although extremely labour intensive, can provide an improved estimate of number mean diameters, if care is taken to ensure that no artefacts are introduced by the sample preparation procedure [34]. The diameters obtained by FCS (Table 2) for the $n \mathrm{SiO}_{2}, n \mathrm{PL}$ and CMC- $n \mathrm{ZVI}$ particles were comparable to those measured by DLS. Also, the FCS-determined $n$ Au and $n$ ZVI diameters were close to their nominal sizes $(10 \mathrm{~nm}$ for $n \mathrm{Au}$ and $77 \mathrm{~nm}$ for $n \mathrm{ZVI})$. Measurements in media $\mathrm{E}$ and $\mathrm{F}$ (data not shown) resulted in sizes of $\sim 5-6 \mathrm{~nm}$, corresponding to the size of albumin [35] which was in very high proportion in comparison with the ENPs (Figure 1).

[TABLE 2 HERE] 
As a complementary characterization tool, TEM was employed to image two ENPs ( $n \mathrm{Au}, \mathrm{QD}$ )

(Figure 3). In PBS (Figure 3i and 3iii), observed particle sizes $(38 \pm 15 \mathrm{~nm}$ for QD and $16 \pm 4$ $\mathrm{nm}$ for the $n \mathrm{Au}$ ) (Table 2) were slightly greater than the nominal particle sizes reported by the manufacturers (10 $\mathrm{nm}$ in each case). DLS results suggested that serum proteins were interacting with the ENP surface and promoting particle stabilization, however these results did not provide information regarding the structure of such protein-stabilized colloids. The mean size of the QD doubled to $65 \pm 18 \mathrm{~nm}$ in the presence of FBS (Figure 3ii) but no particle clusters were observed, thus suggesting adsorption of serum proteins at the QD surface and thereby confirming the DLS observations. From the TEM image (Figure 3iv), the $n \mathrm{Au}$ (black arrows) appeared to not be embedded in clusters of proteins, yielding a mean diameter of $11 \pm 2 \mathrm{~nm}$ for $n \mathrm{Au}$ in FBSsupplemented PBS.

Thus, while there is clear (DLS) evidence for the aggregation of the ENPs in the presence of salts (e.g., PBS), or medium constituents (e.g. sodium pyruvate, L-glutamine) (Figure 1), a substantial number of particles was non-aggregated, which demonstrates a major advantage of using several characterization tools when attempting to understand ENP properties and interactions with proteins.

\section{[FIGURE 3 HERE]}

\section{Discussion}

The main objective of this study was to examine the impact of cell culture media constituents and supplements on ENP stability, by increasing the complexity of the medium composition, starting from simple (ions) to complete cell culture media. Understanding the roles 
of the surface coating on the interactions between ENPs and culture media constituents might help to better understand cytotoxicity results and mechanisms. Theoretically, anionic functional groups on the particle surface should reduce the interaction of ENPs with negatively charged media components such as proteins. But at high salt concentrations, bridging between the proteins and the surface of the ENPs might occur $[15,18,36]$. Earlier studies conducted with nanoparticles stabilized with polymers or polyelectrolytes [17, 37-39] have reported an important electrostatic repulsion between the coated ENPs. Comparisons between these studies suggested that when the particle surface coating was complex, repulsive forces are involved, resulting in decreased interactions between the modified particles and the suspended molecules in the culture medium.

Comparison of the particle diameters in media A and B showed either a small increase in diameter with increasing $I$ or negligible effects. Negative EPMs were generally lower at higher salt concentrations. Such a result is in qualitative agreement with classical colloid theory in which the particle Debye layer is compressed due to the screening of the particle charge. Similar results have been found recently for $n \mathrm{TiO}_{2}[40,41], n \mathrm{ZVI}$ and CMC- $n \mathrm{ZVI}[24]$ and for iron oxide nanoparticles following their incubation for $24 \mathrm{hr}$ in PBS [42].

Analysis of the effect of sodium pyruvate and L-glutamine revealed that these culture media components had a moderate impact on ENP stability, except for $n \mathrm{Au}$, where an increase in aggregation was observed. Since the $n$ Au stock suspension was stabilized using sodium citrate [31], the addition of molecules with similar size and functionality (Figure S1) would be expected to have comparable effects. The observed decrease in particle stabilization is attributed to the fact that sodium pyruvate and L-glutamine have a lower charge density than citrate and are thus expected to be less effective at stabilizing the gold nanoparticles. Since the nitrogen atoms present in L-glutamine may interact more strongly with gold than the carbon atoms present in citrate [31], 
it is possible that L-glutamine might be able to outcompete the citrate, leading to a decrease in particle stability. Accordingly, a 5-fold increase in the diameter of $n \mathrm{Au}$ was observed following the addition of L-glutamine as compared to PBS alone (Figure 1ii).

In the case of the proteins, the results indicated that the BSA and FBS were strongly adsorbed to the ENPs. In the presence of BSA, particle aggregation may have been a direct consequence of the decrease in EPM, leading to lessened electrostatic repulsion between the nanoparticles. Interaction between BSA and ENPs could also increase the likelihood of protein corona formation around the ENPs or ENP entrapment, potentially leading to the aggregate formation reported for all particles that were tested (with the exception of the $n \mathrm{ZVI}$ ). Casals et al. [43] also showed an increase in $n$ Au size with the addition of BSA. Buford et al. [36] reported the presence of large aggregates of $\mathrm{C}_{60}$ particles when comparing suspensions prepared in PBS and BSA-supplemented PBS, though in some cases, BSA seemed to stabilize the $\mathrm{C}_{60}$ fullerene suspensions. Similar studies have demonstrated a stabilizing effect of human serum albumin for both $\mathrm{C}_{60}[44]$ and $n \mathrm{TiO}_{2}$ suspensions [15]. While these latter reports showed an opposite effect to what was observed here; it should be noted that in many studies [15, 16, 36, 43, 44], albuminsupplemented ENP suspensions were either sonicated and analyzed immediately after preparation or mechanically-shaken for several hours followed by the analysis. The results reported here were for ENPs equilibrated for $24 \mathrm{hrs}$ in the target solution. Finally, it should be noted that although albumin was the main serum component, there are over 3700 other proteins in serum that could potentially interact with the ENPs [45].

The decrease in size upon addition of FBS suggests that serum proteins other than albumin have a stabilizing effect on the ENP. Other studies have demonstrated that high affinity and slower-exchanging proteins replace albumin and adsorb at the surface of copolymer particles [20, 
46]. This phenomenon might be occurring in this study. As a result, the assessed ENP sizes were smaller in the presence of FBS than observed for particles exposed to albumin (BSA) alone. The addition of serum proteins has been shown to disaggregate ENPs and to stabilize the nanoparticles $[15,16,33,36,42,43,47,48]$. In agreement with our findings, Petri-Fink et al. [37] observed increased colloidal stability of polymer-coated superparamagnetic iron oxide nanoparticles in the presence of FBS and Jiang et al. [16] showed that other proteins present in serum could play a role in $n \mathrm{TiO}_{2}$ dispersion in cell culture media.

For the most part, hydrodynamic diameters were larger in the DMEM and F-12K suspensions than for measurements made at low I (Figure 1). Previous studies also showed an increase in particle size for iron oxide nanoparticle suspensions [17, 42] when the background solution was changed from DI water to cell culture medium. Dutta et al. [49] also showed greater aggregation of amorphous silica nanoparticles when they were suspended in normal growth medium as compared with water.

One hypothesis of this study was that ENPs with more complex surface modification would exhibit less interaction with medium components. Indeed, the carboxyl-functionalized ENPs (CMC-nZVI and $n \mathrm{PL})$ were less affected by the medium components than the non-stabilized particles ( $n \mathrm{Au}$ and bare $n \mathrm{ZVI})$, and the polymer-embedded ENPs ( $n \mathrm{SiO}_{2}$ and QDs) appeared to have the least interaction with medium components of all the ENP studied. Finally, while several general trends were noted here, it was clear that predictions of colloidal stability in complex media are still likely to be difficult and that particle characterization, specific to a given set of experimental conditions, is likely to be the best manner to obtain a mechanistic understanding of future cytotoxicity studies. 


\section{Conclusions}

Physicochemical characteristics (size and EPM) for a selected set of ENPs were assessed in the presence of selected cell culture media components. Our results show that each examined ENP had a specific affinity for media constituents or supplements, making the generalization of their behavior in cell culture media difficult. For example, two ENPs with comparable nominal size (i.e. QD and $n \mathrm{Au}$ ) did not exhibit the same colloidal stability when suspended with inorganic salt or in the presence of cell media constituents (L-glutamine or sodium pyruvate). Nevertheless, some general trends can be inferred from the current study. Albumin - the major protein found in serum - did not seem to have a stabilizing effect on any of the ENPs studied here. This study shows that possible ENP cytotoxicity cannot be predicted without knowing the influence of cell culture media and serum components on the nanoparticle stability. By categorizing the selected ENPs by level of surface modification, it was shown that the type of ENP surface (non-coated, carboxyl-functionalized, or polymer-embedded) played a significant role in colloidal stability: the complexity of interactions between the ENPs and the medium components generally decreased with increasing complexity of the particle surface. Careful evaluation of the interfacial interactions

via a systematic approach will be necessary in order to thoroughly understand the fundamentals of ENP cytotoxicity. 


\section{Acknowledgements}

This work is supported by the Natural Sciences and Engineering Research Council of Canada, the Fonds Quebecois de la Recherche sur la Nature et les Technologies (FQRNT), l'Institut (Robert

Sauvé) de Recherche sur la Santé et la Sécurité au Travail (IRSST) and the Canada Research Chairs (CRC) program. The authors acknowledge S. J. Brennan, A. R. Petosa and G. Hidalgo (McGill) for assistance with manuscript editing. 


\section{LITERATURE CITED}

[1] C.C. Berry, S. Wells, S. Charles, A.S.G. Curtis, Biomaterials 24 (2003) 4551-4557.

[2] M. Ferrari, Nat. Rev. Cancer 5 (2005) 161-171.

[3] M.C. Roco, Curr. Opin. Biotechnol. 14 (2003) 337-346.

[4] J.L. West, N.J. Halas, Annu. Revi. Biomed. Eng. 5 (2003) 285-292.

[5] S.J. Klaine, P.J.J. Alvarez, G.E. Batley, T.F. Fernandes, R.D. Handy, D.Y. Lyon, S. Mahendra, M.J. McLaughlin, J.R. Lead, Environ. Toxicol. Chem. 27 (2008) 1825-1851.

[6] N. Lewinski, V. Colvin, R. Drezek, Small 4 (2008) 26-49.

[7] A. Nel, T. Xia, L. Madler, N. Li, Science 311 (2006) 622-627.

[8] G. Oberdorster, E. Oberdorster, J. Oberdorster, Environ. Health Perspect. 113 (2005) 823839.

[9] J.S. Chang, K.L.B. Chang, D.F. Hwang, Z.L. Kong, Environ. Sci. Technol. 41 (2007) 20642068.

[10] A.M. Derfus, W.C.W. Chan, S.N. Bhatia, Nano Letters 4 (2004) 11-18.

[11] A. Hoshino, K. Fujioka, T. Oku, M. Suga, Y.F. Sasaki, T. Ohta, M. Yasuhara, K. Suzuki, K. Yamamoto, Nano Letters 4 (2004) 2163-2169.

[12] T.S. Hauck, A.A. Ghazani, W.C.W. Chan, Small 4 (2008) 153-159.

[13] T. Xia, M. Kovochich, M. Liong, J.I. Zink, A.E. Nel, ACS Nano 2 (2008) 85-96.

[14] A.E. Nel, L. Madler, D. Velegol, T. Xia, E.M.V. Hoek, P. Somasundaran, F. Klaessig, V. Castranova, M. Thompson, Nature Materials 8 (2009) 543-557.

[15] Z.E. Allouni, M.R. Cimpan, P.J. Hol, T. Skodvin, N.R. Gjerdet, Colloids Surf., B 68 (2009) 83-87.

[16] J.K. Jiang, G. Oberdorster, P. Biswas, J. . Nanoparticle Res. 11 (2009) 77-89.

[17] B. Chanteau, J. Fresnais, J.F. Berret, Condens. Matter (2009) 1-10, arXiv:0907 $1008 v 0901$.

[18] R. Tantra, J. Tompkins, P. Quincey, Colloids Surf., B 75 (2010) 275-281.

[19] N.B. Saleh, L.D. Pfefferle, M. Elimelech, Environ. Sci. Technol. 44 (2010) 2412-2418.

[20] T. Cedervall, I. Lynch, S. Lindman, T. Berggard, E. Thulin, H. Nilsson, K.A. Dawson, S. Linse, Proc. Natl. Acad. Sci. U.S.A. 104 (2007) 2050-2055.

[21] M. Lundqvist, J. Stigler, G. Elia, I. Lynch, T. Cedervall, K.A. Dawson, Proc. Natl Acad. Sci. U.S.A. 105 (2008) 14265-14270.

[22] R.I. Freshney, Culture of Animal Cells. A Manual of Basic Technique. 2nd Ed, 1987.

[23] List of Manufactured Nanomaterials and List of Endpoints for Phase One of the Sponsorship Program for the Testing of Manufactured Nanomaterials: Revision, in: S.o.t.S.o.M. Nanomaterials (Ed.), 2010.

[24] J. Fatisson, S. Ghoshal, N. Tufenkji, Langmuir 26 (2010) 12832-12840.

[25] R.F. Domingos, M.A. Baalousha, Y. Ju-Nam, M.M. Reid, N. Tufenkji, J.R. Lead, G.G. Leppard, K.J. Wilkinson, Environ. Sci. Technol. 43 (2009) 7277-7284.

[26] E.L. Elson, D. Magde, Biopolymers 13 (1974) 1-27.

[27] K. Starchev, K.J. Wilkinson, J. Buffle, Springer Series in Chemical Physics 65 (2001) 251-

275.

[28] P.-O. Gendron, F. Avaltroni, K.J. Wilkinson, J. of Fluorescence 18 (2008) 1093-1101.

[29] P. Mulvaney, Langmuir 12 (1996) 788-800. 
[30] J. Liao, Y. Zhang, W. Yu, L. Xu, C. Ge, J. Liu, N. Gu, Colloids Surf., A 223 (2003) 177-183.

[31] I. Ojea-Jimenez, V. Puntes, J. Am. Chem. Soc. 132 (2009) 5322.

[32] D. Mavrocordatos, D. Perret, G.G. Leppard, Strategies and Advances in the Characterisation of Environmental Colloids by Electron Microscopy Denis Mavrocordatos, John Wiley \& Sons, Ltd, 2007.

[33] R.C. Murdock, L. Braydich-Stolle, A.M. Schrand, J.J. Schlager, S.M. Hussain, Toxicol. Sci. 101 (2008) 239-253.

[34] G.G. Leppard, Current Nanoscience 4 (2008) 278-301.

[35] X.M. He, D.C. Carter, Nature (London, United Kingdom) 358 (1992) 209-215.

[36] M.C. Buford, R.F. Hamilton, Jr., A. Holian, Particle and Fibre Toxicol. 4 (2007) No pp given.

[37] A. Petri-Fink, B. Steitz, A. Finka, J. Salaklang, H. Hofmann, Europ. J. Pharm. Biopharm. 68 (2008) 129-137.

[38] T. Phenrat, N. Saleh, K. Sirk, H.-J. Kim, R.D. Tilton, G.V. Lowry, J. Nanoparticle Res. 10 (2008) 795-814.

[39] N. Saleh, T. Phenrat, K. Sirk, B. Dufour, J. Ok, T. Sarbu, K. Matyjaszewski, R.D. Tilton, G.V. Lowry, Nano Letters 5 (2005) 2489-2494.

[40] J. Fatisson, R.F. Domingos, K.J. Wilkinson, N. Tufenkji, Langmuir 25 (2009) 6062-6069.

[41] R.F. Domingos, N. Tufenkji, K.J. Wilkinson, Environ. Sci. Technol. 43 (2009) 1282-1286.

[42] Z.P. Chen, Y. Zhang, K. Xu, R.Z. Xu, J.W. Liu, N. Gu, J. Nanosci. Nanotechnol. 8 (2008) 6260-6265.

[43] E. Casals, T. Pfaller, A. Duschl, G.J. Oostingh, V. Puntes, ACS Nano 4 (2010) 3623-3632.

[44] S. Deguchi, T. Yamazaki, S.-a. Mukai, R. Usami, K. Horikoshi, Chem. Res. Toxicol. 20 (2007) 854-858.

[45] B. Muthusamy, G. Hanumanthu, S. Suresh, B. Rekha, D. Srinivas, L. Karthick, B.M. Vrushabendra, S. Sharma, G. Mishra, P. Chatterjee, K.S. Mangala, H.N. Shivashankar, K.N. Chandrika, N. Deshpande, M. Suresh, N. Kannabiran, V. Niranjan, A. Nalli, T.S. Keshava Prasad, K.S. Arun, R. Reddy, S. Chandran, T. Jadhav, D. Julie, M. Mahesh, S. Lynate John, K. Palvankar, D. Sudhir, P. Bala, N.S. Rashmi, G. Vishnupriya, K. Dhar, S. Reshma, R. Chaerkady, T.K.B. Gandhi, H.C. Harsha, S. Sujatha Mohan, K.S. Deshpande, M. Sarker, A. Pandey, Proteomics 5 (2005) 35313536.

[46] T. Cedervall, I. Lynch, M. Foy, T. Berggard, S.C. Donnelly, G. Cagney, S. Linse, K.A. Dawson, Angew. Chem. Int. Ed. 46 (2007) 5754-5756.

[47] B.D. Chithrani, A.A. Ghazani, W.C.W. Chan, Nano Letters 6 (2006) 662-668.

[48] C. Fang, N. Bhattarai, C. Sun, M. Zhang, Small 5 (2009) 1637-1641.

[49] D. Dutta, S.K. Sundaram, J.G. Teeguarden, B.J. Riley, L.S. Fifield, J.M. Jacobs, S.R. Addleman, G.A. Kaysen, B.M. Moudgil, T.J. Weber, Toxicol. Sci. 100 (2007) 303-315. 


\section{Figures and Tables}

\section{Captions}

Table 1. Compositions of the different media used.

Table 2. Summary of measured ENP sizes determined by FCS and TEM for selected ENPs in selected conditions.

Figure 1. Measured EPM (grey bars and left-hand y-axis) and DLS sizes (white bars and right-hand y-axis), for (i) bare $n \mathrm{ZVI}$, (ii) $n \mathrm{Au}$, (iii) CMC-nZVI, (iv) $n \mathrm{PL}$, (v) $n \mathrm{SiO}_{2}$ and (vi) QD in media $\mathrm{A}$ to $M$ (Table 1). Values represent means and confidence intervals (95\%) for each condition, which was measured in triplicate. The symbols $\left({ }^{*}\right.$ or $\star$ and + or $\times$ ) denote a statistically significant difference $(p<0.05)$ in the physicochemical characteristics (size and EPM, respectively) for each condition versus their respective control solution (i.e. ${ }^{*}$ and + were used for conditions $\mathrm{C}-\mathrm{H}, \mathrm{J}$ and $\mathrm{L}$ which were compared to $\mathrm{B}, \star$ and $\times$ were used for conditions $\mathrm{K}$ and $\mathrm{M}$ which were respectively compared to $\mathrm{J}$ and $\mathrm{L}$ ).

Figure 2. UV-Vis spectra of $n$ Au colloid in water (solid line) and in PBS + sodium pyruvate (medium C) (dotted line) measured at $[n \mathrm{Au}]=1.91 \times 10^{12}$ particles $/ \mathrm{mL}$.

Figure 3. Representative TEM images of: (i) QD in PBS, (ii) QD in PBS + FBS $10 \%$, (iii) $n \mathrm{Au}$ in PBS, and (iv) $n \mathrm{Au}$ in PBS + FBS $10 \%$, after 24 hrs incubation at $37^{\circ} \mathrm{C}$. 


\begin{tabular}{|c|c|c|c|c|c|c|c|c|}
\hline \multirow[t]{2}{*}{ Medium } & \multirow[t]{2}{*}{ lonic strength } & \multirow[t]{2}{*}{$\mathrm{pH}$} & \multirow[t]{2}{*}{$\begin{array}{l}\text { Background } \\
\text { medium }\end{array}$} & \multicolumn{3}{|c|}{ Media constituents } & \multicolumn{2}{|c|}{$\begin{array}{l}\text { Serum protein } \\
\text { supplements }\end{array}$} \\
\hline & & & & Constituent & Concentration & Type & Name & Concentration \\
\hline$A$ & $1 \mathrm{mM}$ & 8.3 & Water & sodium bicarbonate & $1 \mathrm{mM}$ & $\begin{array}{c}\text { Inorganic } \\
\text { salt }\end{array}$ & & \\
\hline$B$ & $\sim 150 \mathrm{mM}$ & 7.4 & PBS & & & & & \\
\hline C & $\sim 150 \mathrm{mM}$ & 7.4 & PBS & sodium pyruvate & $1 \mathrm{mM}$ & Organic salt & & \\
\hline$D$ & $\sim 150 \mathrm{mM}$ & 7.4 & PBS & L-glutamine & $2 \mathrm{mM}$ & Amino acid & & \\
\hline$E$ & $\sim 150 \mathrm{mM}$ & 7.4 & PBS & & & & Albumin (BSA) & $0.1 \%$ \\
\hline $\mathrm{F}$ & $\sim 150 \mathrm{mM}$ & 7.4 & PBS & & & & Albumin (BSA) & $10 \%$ \\
\hline G & $\sim 150 \mathrm{mM}$ & 7.4 & PBS & & & & Serum (FBS) & $0.1 \%$ \\
\hline $\mathrm{H}$ & $\sim 150 \mathrm{mM}$ & 7.4 & PBS & & & & Serum (FBS) & $10 \%$ \\
\hline $\mathrm{J}$ & $\sim 150 \mathrm{mM}$ & 7.4 & DMEM & see Table S1 & & $\begin{array}{l}\text { Cell culture } \\
\text { medium }\end{array}$ & & \\
\hline $\mathrm{K}$ & $\sim 150 \mathrm{mM}$ & 7.4 & DMEM & see Table S1 & & $\begin{array}{l}\text { Cell culture } \\
\text { medium }\end{array}$ & Serum (FBS) & $10 \%$ \\
\hline $\mathrm{L}$ & $\sim 150 \mathrm{mM}$ & 7.4 & F12-K & see Table S1 & & $\begin{array}{l}\text { Cell culture } \\
\text { medium }\end{array}$ & & \\
\hline$M$ & $\sim 150 \mathrm{mM}$ & 7.4 & F12-K & see Table S1 & & $\begin{array}{l}\text { Cell culture } \\
\text { medium }\end{array}$ & Serum (FBS) & $10 \%$ \\
\hline
\end{tabular}

FATISSON et al

TABLE 1

\begin{tabular}{|c|c|c|c|c|c|c|c|}
\hline Medium & $n \mathrm{SiO}_{2}$ & Bare $n Z \mathrm{VI}$ & CMC-nZVI & $n P L$ & \multicolumn{2}{|c|}{$n A u$} & QD \\
\hline & \multicolumn{5}{|c|}{ FCS Size $(\mathrm{nm})$} & \multicolumn{2}{|c|}{ TEM Size $(\mathrm{nm})$} \\
\hline A & $117 \pm 18$ & $51 \pm 20$ & $51 \pm 22$ & $47 \pm 13$ & $20 \pm 5$ & & \\
\hline B & $130 \pm 47$ & $77 \pm 32$ & $47 \pm 36$ & $50 \pm 17$ & $23 \pm 8$ & $16 \pm 4$ & $38 \pm 15$ \\
\hline D & $83 \pm 13$ & $62 \pm 26$ & $105 \pm 48$ & $52 \pm 3$ & $23 \pm 10$ & & \\
\hline $\mathrm{H}$ & $178 \pm 49$ & $124 \pm 32$ & $72 \pm 27$ & $49 \pm 8$ & $33 \pm 1$ & $11 \pm 2$ & $65 \pm 18$ \\
\hline $\mathrm{J}$ & $94 \pm 18$ & $55 \pm 40$ & $46 \pm 11$ & $72 \pm 27$ & $22 \pm 6$ & & \\
\hline K & $134 \pm 49$ & $148 \pm 67$ & $107 \pm 74$ & $82 \pm 28$ & $39 \pm 0$ & & \\
\hline
\end{tabular}

FATISSON et al

TABLE 2 

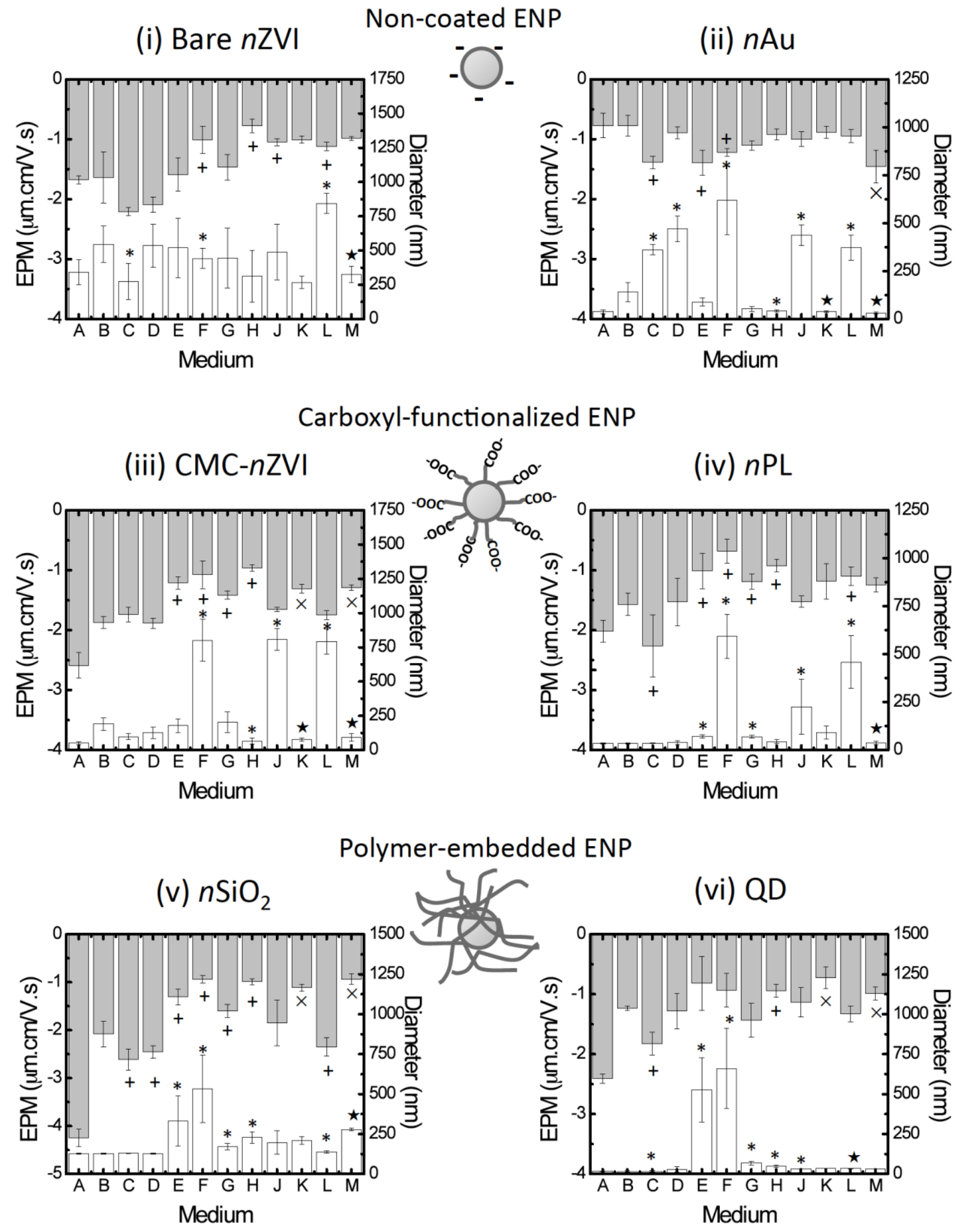

FATISSON et al FIGURE 1 


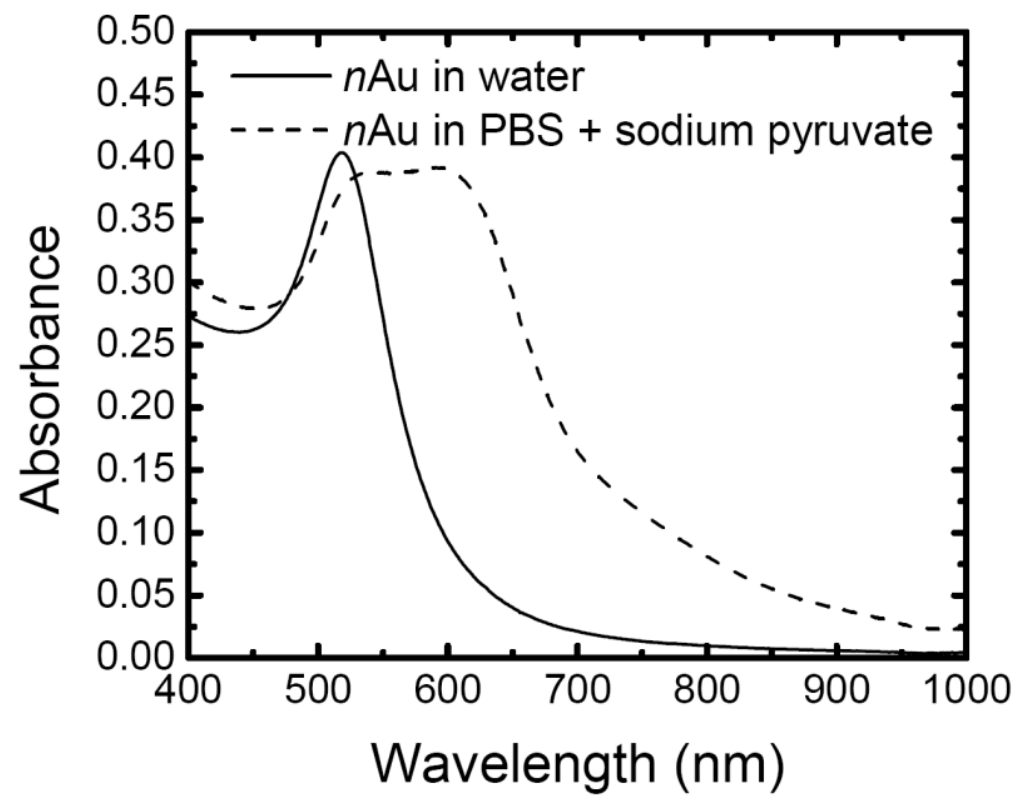

FATISSON et al FIGURE 2 

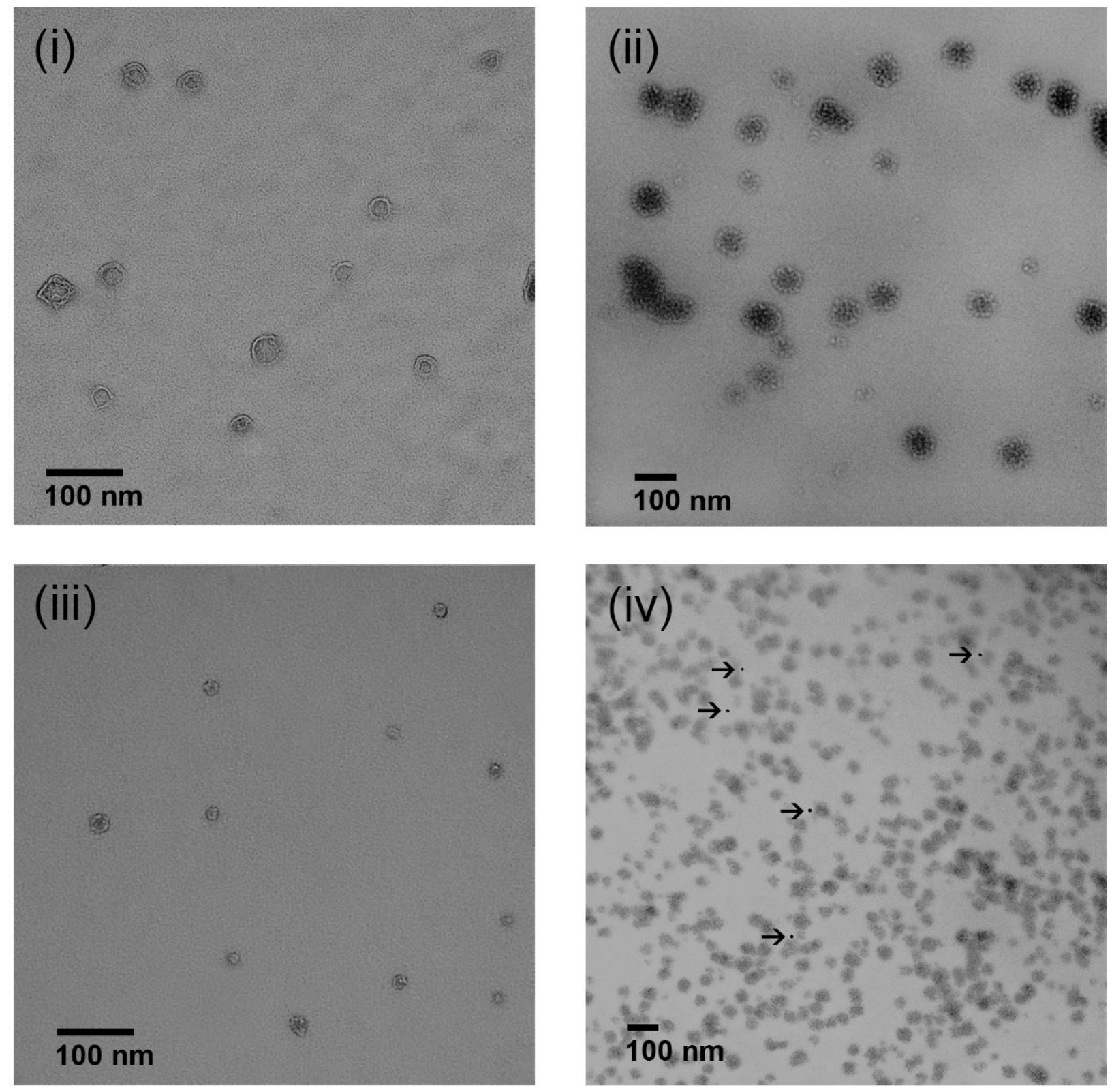

FATISSON et al FIGURE 3 